\title{
Analysis and Optimization of the Performance of OFDM on Frequency-Selective Time-Selective Fading Channels
}

\author{
Heidi Steendam and Marc Moeneclaey, Senior Member, IEEE
}

\begin{abstract}
In mobile radio communication, the fading channels generally exhibit both time-selectivity and frequency-selectivity. Orthogonal frequency-division multiplexing has been proposed to combat the frequency-selectivity, but its performance is also affected by the time-selectivity. In this paper, we investigate how various parameters, such as the number of carriers, the guard time length, and the sampling offset between receiver and transmitter, affect the system performance. Further, we determine the optimum values of the above parameters, which minimize the degradation of the signal-to-noise ratio at the input of the decision device.
\end{abstract} tion.

Index Terms-Fading channel, OFDM, performance optimiza-

\section{INTRODUCTION}

D UE TO the enormous growth of wireless services (cellular telephones, wireless LAN's, etc.) during the last decade, the need of a modulation technique that can transmit reliably high data rates at a high bandwidth efficiency arises [1]-[3]. In a mobile radio channel, the signal is disturbed by multipath fading which generally exhibits both time-selectivity and frequency-selectivity. The signal power is carried by a large number of paths with different strengths and delays. For the global system for mobile telecommunications, typical multipath intensity profiles are defined for rural areas (RA), urban areas [typical urban area (TU)], and hilly terrain areas (HT) [4], [5].

The influence of the intersymbol interference (ISI) caused by the frequency-selectivity can be reduced by increasing the duration of a transmitted symbol. This can be accomplished by using orthogonal frequency-division multiplexing (OFDM). The symbol sequence to be transmitted is split into a large number of lower speed symbol streams, where each modulates a different carrier. The carrier spacing is selected such that modulated carriers are orthogonal over a symbol interval. In addition, a guard interval (cyclic prefix) is inserted in order to combat the frequency-selectivity of the channel [6], [7]. The transmitter and receiver for OFDM can be implemented efficiently by using fast Fourier transform techniques. OFDM

Paper approved by Y. Li, the Editor for Wireless Communication Theory of the IEEE Communications Society. Manuscript received May 6, 1998; revised February 15, 1999. This paper was presented in part at the International Symposium on Signals, Systems, and Electronics (ISSE), Pisa, Italy, 1998.

The authors are with the Communications Engineering Laboratory, University of Gent, B-9000 Gent, Belgium (e-mail: marc.moeneclaey@ telin.rug.ac.be).

Publisher Item Identifier S 0090-6778(99)09773-1. has been proposed and/or accepted for various applications, such as broadcasting of digital audio and digital television [8], mobile radio [1], [2], [9]-[11], and transmission over twisted pair cables (asymmetric digital subscriber line) [12], [13].

Increasing the duration of a transmitted symbol, however, makes the system more sensitive to the time-selectivity of the channel. As the time-selectivity affects the orthogonality of the carriers, a larger symbol duration gives rise to more intercarrier interference (ICI). The lengthening of the symbol duration, introduced to combat the frequency-selectivity, therefore is limited by the time-selectivity.

In [10] and [11], the number of subchannels of the OFDM system is optimized in the case of a sufficient guard interval, i.e., the guard interval is longer than the delay spread. In this contribution, we investigate the effect of an insufficient guard interval as well as the effect of the number of carriers and the sampling offset between receiver and transmitter on the performance of the OFDM system. The OFDM system is described in Section II. In Section III, we consider the degradation of the signal-to-noise ratio (SNR) at the input of the decision device as performance measure of the OFDM system. Section IV focuses on two limiting cases, i.e., the time-flat channel (for a small number of carriers) and the frequency-flat channel (for a large number of carriers), and analyzes the resulting interference powers. The problem of frame synchronization is considered in Section V. Numerical results, including performance optimizations, are presented in Section VI. Finally, conclusions are drawn in Section VII.

\section{SYSTEM DESCRIPTION}

In OFDM, the available bandwidth is partitioned into $N$ subchannels that are made orthogonal by using carriers with a spacing equal to the subchannel symbol rate. A binary message is coded and mapped to a sequence of complex data symbols, which are split into frames of $N$ symbols: $a_{i, n}$ denotes the $n$th symbol of the $i$ th frame $(0 \leq n \leq N-1,-\infty<i<+\infty)$. The $n$th carrier is modulated by the symbols $\left\{a_{i, n} \mid-\infty<\right.$ $i<+\infty\}$, and the modulated carriers are summed before transmission. In a practical implementation, the $N$ samples of the transmitted signal corresponding to the $i$ th frame are generated by feeding $\left\{a_{i, n} \mid n=0, \cdots, N-1\right\}$ to an inverse discrete Fourier transform (DFT) (see Fig. 1). The loss of orthogonality between the carriers, caused by the dispersive channel, introduces ISI and ICI at the receiver. To combat 


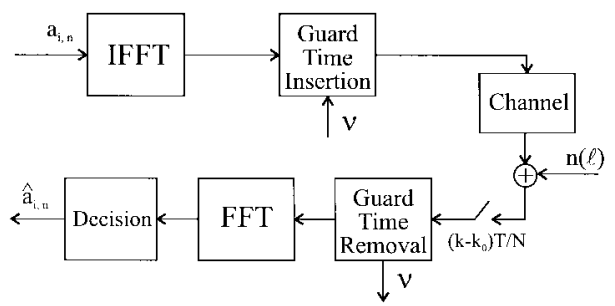

Fig. 1. An OFDM transceiver.

this interference, each frame is preceded by a guard interval of $\nu$ samples containing a cyclic extension of the transmitted time-domain samples (cyclic prefix). The $i$ th transmitted frame (including the prefix) contains $N+\nu$ time-domain samples, of which the $m$ th sample is given by

$$
\begin{array}{r}
g_{i}(m)=\sqrt{\frac{E_{s}}{N+\nu}} \sum_{n=0}^{N-1} a_{i, n} e^{j 2 \pi((n m) / N)}, \\
m=-\nu, \cdots, N-1 .
\end{array}
$$

Assuming the data symbols are statistically independent and have a unit average energy, i.e., $E\left[a_{i, n} a_{j, k}^{*}\right]=\delta_{i, j} \delta_{n, k}$, the transmitted average energy per symbol equals $E_{s}$.

Many wireless communication channels can be modeled as multipath Rayleigh fading channels having an impulse response $h(k ; \ell)$, represented by a tapped delay line where the $k$ th coefficient is a Gaussian random process with time variable $\ell$. Bello [4] introduced the wide-sense stationary uncorrelated scattering (WSSUS) model to easily describe fading channels. This model, which is valid for most radio channels, assumes that the signal variations arriving at different delays are uncorrelated and that the correlation properties of the channel are stationary. The autocorrelation function, considering these assumptions, yields

$$
E\left[h\left(k_{1}, \ell_{1}\right) h^{*}\left(k_{2}, \ell_{2}\right)\right]=\delta\left(k_{1}-k_{2}\right) R\left(k_{1} ; \ell_{1}-\ell_{2}\right) .
$$

The channel, having an autocorrelation function $R(k ; \ell)$, can be characterized by a multipath intensity profile $R(k ; 0)$ and a Doppler spectrum $S_{D}\left(e^{j 2 \pi f T}\right)$, with

$$
S_{D}\left(e^{j 2 \pi f T}\right)=\sum_{\ell=-\infty}^{+\infty} \sum_{k=-\infty}^{+\infty} R(k ; \ell) e^{-j 2 \pi f \ell T} .
$$

Without loss of generality, we assume that the largest value of the multipath intensity profile occurs at $k=0$, i.e., $R(0 ; 0) \geq R(k ; 0)$. In addition, the received signal is corrupted by complex-valued additive white Gaussian noise (AWGN) with a power spectral density $N_{0}$.

For each transmitted frame of $N+\nu$ samples, the receiver selects $N$ consecutive samples to be processed further and drops the other $\nu$ samples (guard time removal). The indices of the $N$ remaining samples corresponding to the $j$ th frame are $\left\{k-k_{0}+j(N+\nu) \mid k=0, \cdots, N-1\right\}$. The sampling offset $k_{0}$ is assumed to be provided by a frame synchronization algorithm, which selects $k_{0}$ such that the SNR at the input of the decision device is maximum. The remaining samples $r(k)$ of the $j$ th frame, given by

$$
\begin{aligned}
r(k) & =\sum_{i=-\infty}^{+\infty} \sum_{m=-\nu}^{N-1} g_{i}(m) h(k-m-i(N+\nu) ; k)+n(k) \\
k & =-k_{0}+j(N+\nu), \cdots,-k_{0}+j(N+\nu)+N-1
\end{aligned}
$$

are demodulated using a DFT. Each of the $N$ outputs of the DFT is scaled and rotated (single-tap equalization per DFT output) and applied to the decision device.

\section{System Performance}

In the following, we concentrate on the detection of the data symbols during the frame $j=0$. Due to the loss of orthogonality caused by the fading channel, the outputs of the DFT are disturbed by interference, which adds to the channel noise. The power $P(n)$ at the $n$th output of the DFT can be decomposed as

$$
P(n)=E_{s} \frac{N}{N+\nu}\left(P_{U}(n)+P_{\mathrm{ICI}}(n)+P_{\mathrm{ISI}}(n)\right)+N_{0} .
$$

The useful power $P_{U}$ denotes the contribution from the symbol $a_{0, n}$. The ICI power $P_{\text {ICI }}$ contains the contributions from the other symbols transmitted in the considered frame $(i=0)$, whereas the ISI power $P_{\text {ISI }}$ contains the contributions from all symbols transmitted in other frames $(i \neq 0)$. Finally, $N_{0}$ denotes the contribution from the additive noise. Assuming all $N$ carriers are modulated, one obtains

$$
\begin{aligned}
P_{U}(n) & =E\left[\left|\gamma_{n, n, 0}\left(k_{0}\right)\right|^{2}\right] \\
P_{\mathrm{ICI}}(n) & =\sum_{\substack{\ell=0 \\
\ell \neq n}}^{N-1} E\left[\left|\gamma_{\ell, n, 0}\left(k_{0}\right)\right|^{2}\right] \\
P_{\mathrm{ISI}}(n) & =\sum_{\substack{i=-\infty \\
i \neq 0}}^{+\infty} \sum_{\ell=0}^{N-1} E\left[\left|\gamma_{\ell, n, i}\left(k_{0}\right)\right|^{2}\right]
\end{aligned}
$$

where $\gamma_{\ell, n, i}\left(k_{0}\right)$, given by

$$
\begin{aligned}
\gamma_{\ell, n, i}\left(k_{0}\right)=\frac{1}{N} \sum_{m=-\nu}^{N-1} \sum_{\substack{k=0 \\
\cdot h\left(k-k_{0}-m-i(N+\nu) ; k-k_{0}\right)}}^{N-1} e^{-j 2 \pi((k \ell-n m) / N)} &
\end{aligned}
$$

denotes the signal component at the $n$th DFT output during the frame $j=0$, caused by the symbol $a_{\ell, i}$ which is transmitted on the $\ell$ th carrier during the $i$ th frame. It can be verified that in the case of all carriers modulated, the useful power $P_{U}(n)$ and the interference powers $P_{\mathrm{ICI}}(n)$ and $P_{\mathrm{ISI}}(n)$ are independent of the considered carrier. In the following, we can therefore omit the carrier index $n$.

The SNR at the output of the DFT is defined as the ratio of the power of the useful component to the power of the remaining contributions

$$
\mathrm{SNR}=\frac{E_{s} \frac{N}{N+\nu} P_{U}}{E_{s} \frac{N}{N+\nu}\left(P_{\mathrm{ICI}}+P_{\mathrm{ISI}}\right)+N_{0}} .
$$




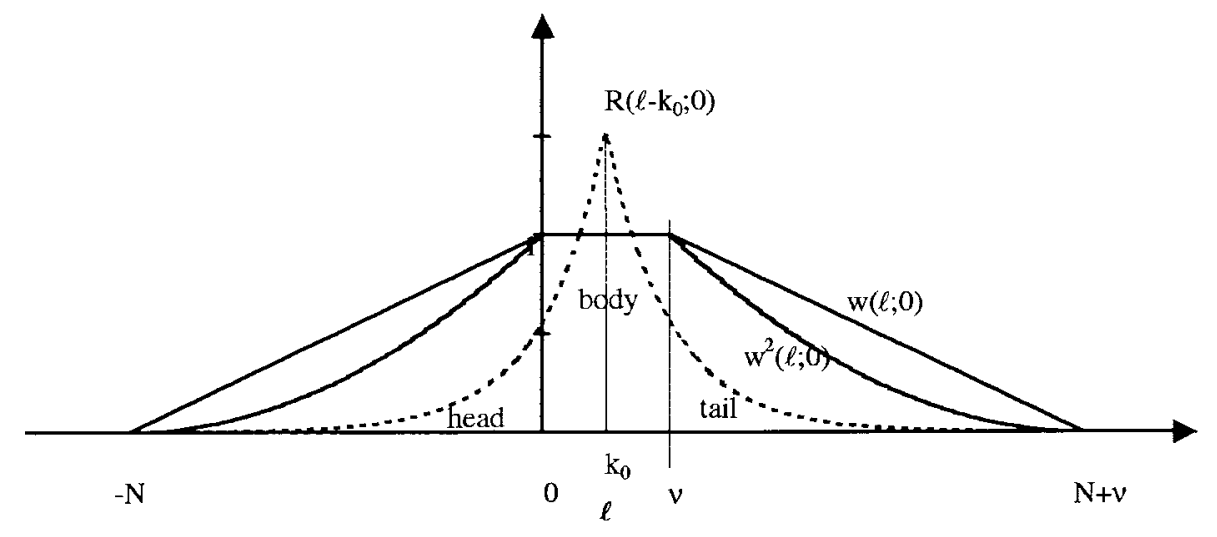

Fig. 2. The weight function $w(k ; 0)$.

In the presence of the fading channel, the SNR is reduced as compared to the case of an AWGN channel. The AWGN channel yields $P_{U}=1, P_{\mathrm{ICI}}=P_{\mathrm{ISI}}=0$, so that for $\nu=0$ the SNR equals $E_{s} / N_{0}$. Assuming the impulse response $h(k ; \ell)$ has a unit average energy (i.e., $\sum_{k=-\infty}^{+\infty} R(k ; 0)=1$ ), it can be verified that the sum of the useful power and the interference powers is given by $P_{U}+P_{\mathrm{ICI}}+P_{\mathrm{ISI}}=1$ (see Appendix A). Under these considerations, the degradation of the SNR expressed in decibels is given by

$$
\operatorname{Deg}=-10 \log \left(\frac{\frac{N}{N+\nu} P_{U}}{1+\frac{E_{S}}{N_{0}} \frac{N}{N+\nu}\left(1-P_{U}\right)}\right) .
$$

For large $E_{s} / N_{0}$, the SNR (8) is limited by $P_{U} /\left(1-P_{U}\right)$ which indicates that the performance is limited by the interference. Hence, increasing $E_{s} / N_{0}$ far beyond $\left(\left(1-P_{U}\right) N /(N+\nu)\right)^{-1}$ yields only a marginal performance improvement.

In a further analysis of the powers in (6), taking into account the above-mentioned considerations, it can be verified that (see Appendix A)

$$
\begin{aligned}
P_{U} & =\frac{1}{N} \sum_{k=-\infty}^{+\infty} \sum_{\ell=-\infty}^{+\infty} w(k ; \ell) R\left(k-k_{0} ; \ell\right) \\
P_{\mathrm{ICI}} & =\sum_{k=-\infty}^{+\infty} w(k ; 0) R\left(k-k_{0} ; 0\right)-P_{U} \\
P_{\mathrm{ISI}} & =\sum_{k=-\infty}^{+\infty}(1-w(k ; 0)) R\left(k-k_{0} ; 0\right)
\end{aligned}
$$

where $w(k ; \ell)$ is a two-dimensional weight function (A2), and $w(k ; 0)$ is shown in Fig. 2. According to (10), the ISI power $P_{\mathrm{ISI}}$ is independent of the time correlation properties of the channel; taking into account the weight function $w(k ; 0)$ from Fig. 2, it follows that $P_{\text {ISI }}$ is determined only by the tails of the multipath intensity profile of the fading channel. The ICI consists of two contributions. The first contribution is independent of the time correlation properties of the fading channel and is mainly determined by the central part (the 'body') of the multipath intensity profile; the second contribution $\left(-P_{U}\right)$ depends on both the dispersive and time-correlation characteristics of the fading channel.

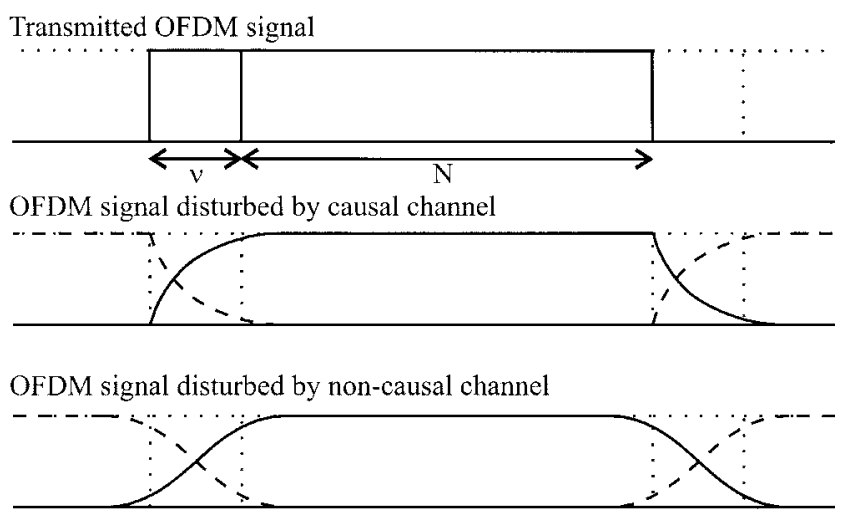

Fig. 3. The OFDM signal.

Let us consider quantitatively the effect of the system parameters (i.e., the guard time length $\nu$ and the number of carriers $N$ ) on the performance degradation (9) as follows.

- For given $N$, increasing $\nu$ reduces the amount of channel distortion on the $N$ samples that are kept by the receiver for further processing (see Fig. 3). Hence, increasing $\nu$ reduces $P_{\mathrm{ICI}}$ and $P_{\mathrm{ISI}}$, so that $P_{U}$ moves closer to 1 . On the other hand, increasing $\nu$ reduces the power efficiency through the factor $N /(N+\nu)$, because the receiver keeps only $N$ of the $N+\nu$ received samples. Note that for an AWGN channel with $\nu>0$, the degradation (9) becomes $10 \log (N /(N+\nu))$, which reflects the power efficiency loss caused by the guard interval.

- For given $\nu$, the dispersive channel introduces a given amount of linear distortion, which is basically confined to a few samples at the edges of the block of $N$ samples that are processed by the receiver. Increasing $N$ reduces the relative importance of these distorted samples, and in addition, increases the power efficiency. On the other hand, increasing $N$ makes the system more sensitive to the timeselectivity of the channel, because the transmitted frames get longer. The time-selectivity affects the orthogonality of the transmitted frames, and therefore, introduces ICI at the DFT output.

From the above considerations, it follows that an optimum set $(\nu, N)$ exists, which minimizes the degradation (9). 


\section{Limiting CASES: Time-Flat ChanNEL AND FREQUENCY-FLAT CHANNEL}

The impulse response of a mobile radio channel generally exhibits a delay spreading as well as a Doppler spreading [4]. The delay spreading originates from the multitude of propagation paths taken by the transmitted signals. The coherence bandwidth, i.e., the minimum bandwidth for which time-dispersion is observable, is inversely proportional to the delay spreading. The variation of the mobile radio channel in time is expressed by the Doppler spreading. The coherence time, corresponding to the minimum signal duration for which distortion becomes noticeable, is inversely proportional to the Doppler spreading. In this section, the system is evaluated for two limiting cases: the time-flat channel and the frequency-flat channel.

When the duration of a transmitted frame is small as compared to the coherence time of the fading channel, the variation in time of the channel during a frame can be neglected: the channel can be approximated by a time-flat channel with $R(k ; \ell)=R(k ; 0)$. Considering the relationship between the coherence time and the Doppler spreading, this timeflat channel approximation corresponds to a fading channel with a Doppler spreading, which is small as compared to the carrier spacing. As the Doppler spreading is proportional to the velocity of the vehicle [14], [15], the time-flat channel can be used to model slowly moving vehicles. For a time-flat channel, the general expression (10a) of the power $P_{U}$ simplifies to

$$
P_{U}=\sum_{k=-\infty}^{+\infty} w^{2}(k ; 0) R\left(k-k_{0} ; 0\right)
$$

where we have taken into account that $(1 / N) \sum_{\ell=-\infty}^{+\infty} w(k ; \ell)$ $=w^{2}(k ; 0)$. Taking into account that $P_{\mathrm{IST}}+P_{\mathrm{ICI}}=1-P_{U}$, it follows from (11) that

$$
P_{\mathrm{ISI}}+P_{\mathrm{ICI}}=\sum_{k=-\infty}^{+\infty}\left(1-w^{2}(k ; 0)\right) R\left(k-k_{0} ; 0\right) .
$$

Considering the nature of $w(k ; 0),(11)$ and (12) indicate that the useful power is mainly determined by the 'body' of the autocorrelation function, while the total interference power only involves the head and tail of $R(k ; 0)$ (see Fig. 2).

Assuming the tails of the multipath intensity profile are much shorter than $N$ samples, (12) is well approximated by

$$
P_{\mathrm{ISI}}+P_{\mathrm{ICI}} \cong \frac{2}{N} \sum_{k=0}^{+\infty} k\left(R\left(-k_{0}-k ; 0\right)+R\left(\nu-k_{0}+k ; 0\right)\right)
$$

which indicates that the total interference is proportional to $1 / N$. In addition, it follows from (13) that the total interference decreases with increasing $\nu$, in a way which depends on the shape of the tail of the multipath intensity profile. Under the same assumptions as above, the ISI power (10c) is well approximated by

$$
\left.P_{\mathrm{ISI}} \cong \frac{1}{N} \sum_{k=0}^{+\infty} k\left(R\left(-k_{0}-k ; 0\right)\right)+R\left(\nu-k_{0}+k ; 0\right)\right) .
$$

Comparing (13) and (14), we conclude that ISI and ICI equally contribute to the interference when the time variations of the channel can be ignored.

When $N$ increases for a given $\nu$ and a given channel autocorrelation function, the number of samples affected by the channel dispersion is small as compared to the total number $(N)$ of samples that are processed per frame; the effect of the channel dispersion becomes negligible. When the frame duration becomes comparable to the coherence time of the channel, the effect of the time variations of the channel becomes noticeable; the total interference (ISI + ICI) increases with $N$. The ISI power (10c) is independent of the coherence time of the channel and is proportional to $1 / N$ when $N$ is much larger that the duration of the tails of the multipath intensity profile [see (14)]. Hence, the total interference is mainly ICI when the time variations of the channel become dominant.

When the frame duration is large as compared to the coherence time and the delay spread, it is shown in Appendix B that the fading channel can be approximated by a frequencyflat channel with $R(k ; \ell)=\tilde{R}(\ell) \delta(k)$, where

$$
\tilde{R}(\ell)=\sum_{k=-\infty}^{+\infty} R(k ; \ell) .
$$

Hence, the frequency-flat model corresponds to a single coefficient $\tilde{h}(0 ; \ell)$ with the same Doppler spectrum as the actual channel. The resulting useful power $P_{U}$ is given by

$$
P_{U}=\frac{1}{N} \sum_{\ell=-N}^{+N}\left(1-\frac{|\ell|}{N}\right) \tilde{R}(\ell) .
$$

\section{FRAME SYNCHRONIZATION}

The receiver makes a detection of the symbols $\left\{a_{0, n} \mid\right.$ $n=0, \cdots, N-1\}$ by processing the samples $\left\{r\left(m-k_{0}\right) \mid\right.$ $m=0, \cdots, N-1\}$. The sampling offset $k_{0}$ determines how much these samples are affected by the channel dispersion and should be selected such that the degradation (9) is minimal. As $P_{U}+P_{\mathrm{ISI}}+P_{\mathrm{ICI}}=1$, minimizing the degradation (9) is equivalent to minimizing the interference $P_{\mathrm{ISI}}+P_{\mathrm{ICI}}$ or maximizing the useful power $P_{U}$.

As the impact of the channel dispersion increases when the frame gets shorter, we will determine the optimum sampling offset $k_{0}$ under the assumption that the frame duration is much less than the coherence time of the channel; hence, the timeflat channel model applies. The resulting $k_{0}$ might no longer be optimum when the frame length is in the order of the coherence time; however, in this case the value of $k_{0}$ is less critical, because the effect of channel dispersion is less important than the effect of the time variation of the channel.

When the time-flat model applies, the optimum value $k_{0}$ minimizes the interference power given by (12). In most cases of practical interest, $N$ is much larger than the tails of the multipath intensity profile, so that minimizing (12) is essentially equivalent to minimizing (13). Minimization of (13) yields an optimum value of $k_{0}$ that does not depend on $N$. Noting that the maximum of the intensity profile $R(k ; 0)$ 
occurs at $k=0$, the optimum value of $k_{0}$ is easily determined in the following cases.

- For causal channels (i.e., $R(k ; 0)=0$ for $k<0$ ), the optimum value of $k_{0}$ is $k_{0}=0$.

- For anticausal channels (i.e., $R(k ; 0)=0$ for $k>0$ ), the optimum value of $k_{0}$ is $k_{0}=\nu$.

- For symmetric channels (i.e., $R(k ; 0)=R(-k ; 0)$ ), the optimum value of $k_{0}$ is $k_{0}=\nu / 2$.

In other cases, the optimum value of $k_{0}$ has to be obtained by numerically minimizing (12) or (13).

In practice, a frame synchronization algorithm should provide the value of $k_{0}$ to the receiver. A suitable frame synchronization algorithm could perform measurements of the multipath intensity profile $R(k ; 0)$, use these measurements in (12) or (13) as if they were the correct values, and finally minimize the resulting (12) or (13). For convenience of implementation, the function to be minimized could be replaced by the area under the tails of $R(k ; 0)$

$$
\operatorname{Area}\left(k_{0}\right)=\sum_{k=0}^{+\infty}\left(R\left(-k_{0}-k ; 0\right)+R\left(\nu-k_{0}+k ; 0\right)\right) \text {. }
$$

A similar frame synchronization mechanism has been proposed in [16], in the context of multicarrier transmission over the twisted pair cable.

\section{NUMERICAL RESULTS}

In the computations, a $5-\mathrm{MHz}$ channel bandwidth and a 1-GHz carrier frequency have been assumed. The Doppler spreading for a typical outdoor radio channel can be calculated straightforwardly from the expression $f_{D}=(v / c) f_{C}, v$ representing the velocity of the mobile $(135 \mathrm{~km} / \mathrm{h}), c$ the velocity of light, and $f_{C}$ the center frequency of the mobile radio channel $(1 \mathrm{GHz})$. The resulting coherence time $T_{0}$, according to the rule of thumb $T_{0}=0.5 / f_{D}$ [14], [15], equals $4 \mathrm{~ms}$. In the literature [4], [5], typical channel impulse responses for various environments are defined. For a TU area, a delay spread of $5 \mu \mathrm{s}$ is taken. Considering the $5-\mathrm{MHz}$ channel bandwidth, it follows that the duration of a sample is $0.2 \mu \mathrm{s}$. The proposed autocorrelation function exhibits an exponentially decaying multipath intensity profile and a Gaussian time correlation profile

$$
\begin{aligned}
R(k ; \ell)=C \exp \left(-\frac{k}{y_{0}}\right) \exp & \left(-\frac{\ell^{2}}{2 \sigma_{0}^{2}}\right) \\
k & \geq 0, \quad-\infty<\ell<+\infty
\end{aligned}
$$

where $C$ is a constant of normalization. Defining the delay spread as the time at which the multipath intensity profile falls $20 \mathrm{~dB}$ below the level of the strongest component, the parameter $y_{0}$ is found to be about five samples. The coherence time $T_{0}$ is fixed to the duration of twice the spreading of the Gaussian time correlation profile yielding $\sigma_{0}=10000$ samples.

In Figs. 4 and 5, we compare the total interference power $P_{\mathrm{ISI}}+P_{\mathrm{ICI}}$ for the following cases:

1) the frequency-selective time-selective channel with autocorrelation function $R(k ; \ell)$ from (18);

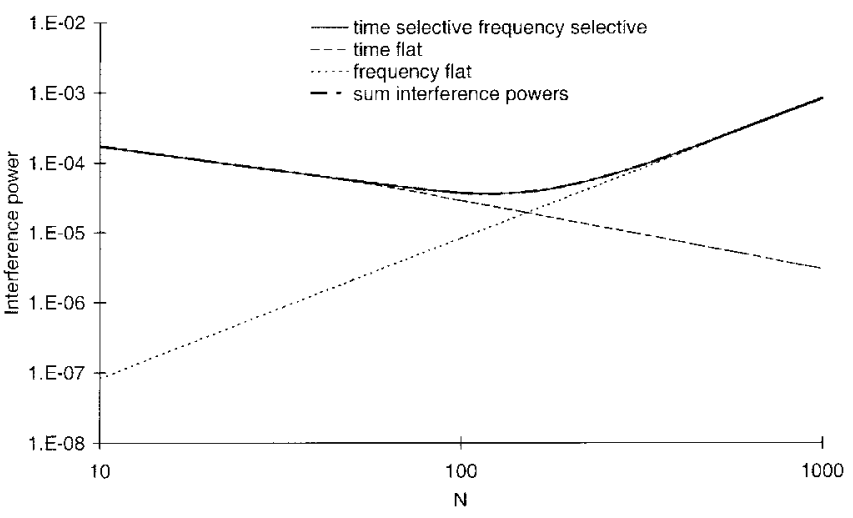

Fig. 4. Interference power as a function of $N(\nu=40)$.

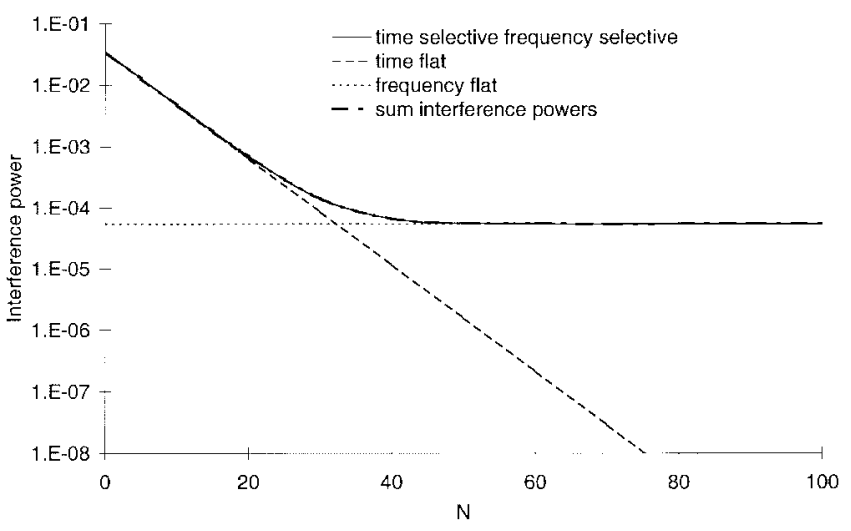

Fig. 5. Interference power as a function of $\nu(N=256)$.

2) the limiting case of the time-flat channel with autocorrelation function $R(k ; 0)$;

$3)$ the limiting case of the frequency-flat channel with autocorrelation function $\tilde{R}(\ell) \delta(k)$, with $\tilde{R}(\ell)$ given by (15); and

4) the sum of the total interference powers resulting from 2) and 3).

Fig. 4 shows the total interference power for $\nu=40$ as a function of $N$, whereas Fig. 5 shows the total interference power for $N=256$ as a function of $\nu$.

We observe from Fig. 4 that the total interference power for the limiting cases of the time-flat channel (2) and the frequency-flat channel (3) converge to the total interference power of the frequency-selective time-selective channel (1), for small $N$ and large $N$, respectively. The total interference power for the time-flat channel is proportional to $1 / N$; this agrees with the result (13). Note that the total interference power for the frequency-flat channel already approaches the total interference power for the frequency-selective timeselective channel, for values of the frame duration that are considerably less than the coherence time of the channel. The resulting total interference power is proportional to $N^{2}$. Finally, we have added the total interference power resulting from the time-flat channel (2) and the frequency-flat channel (3). The resulting sum (4) turns out to be an accurate approximation of the total interference power for the actual frequency-selective time-selective channel (1). Note that the approximation (4) involves the computation of only two single 


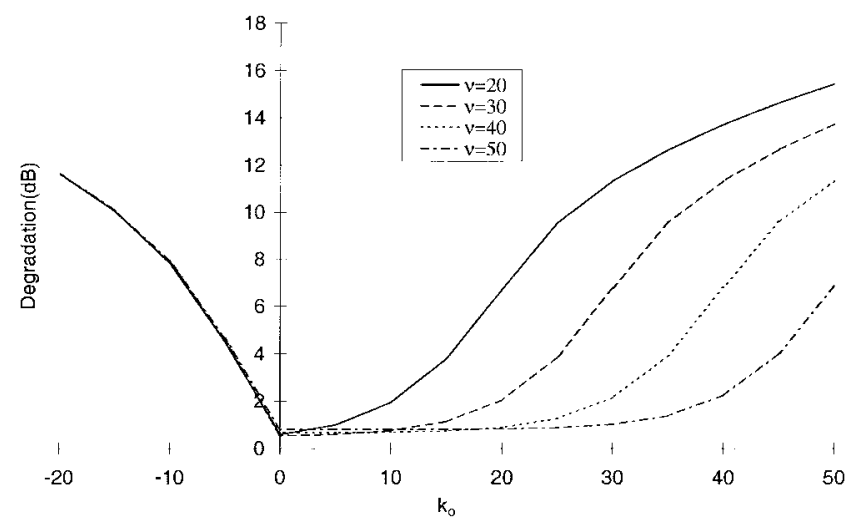

Fig. 6. Degradation as a function of $k_{0}$ : asymmetric profile.

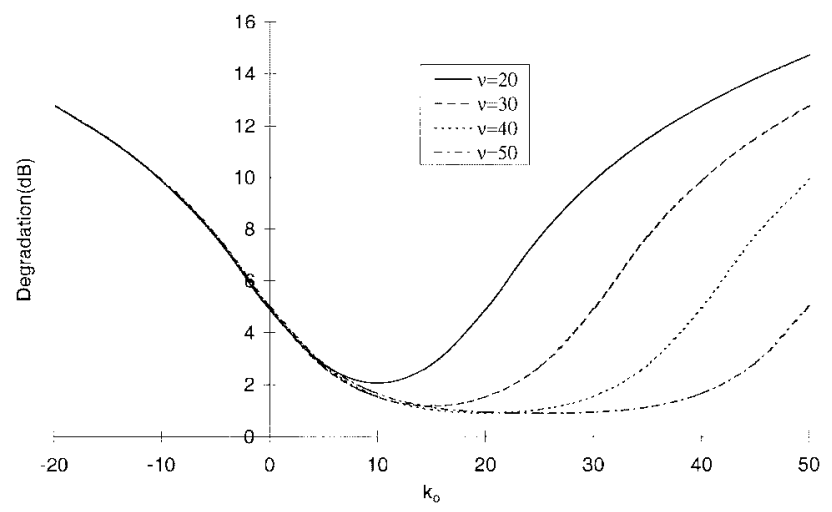

Fig. 7. Degradation as a function of $k_{0}$ : symmetric profile.

summations [i.e., (12) and (16)], whereas the correct result (1) requires a double summation [i.e., (10a)]. Hence, for large $N$, the computation of the approximation is much more efficient than the computation of the correct result.

Fig. 5 shows that for the time-flat channel, the total interference power decreases with increasing $\nu$; this is because the effect of channel dispersion is reduced by increasing the guard interval. This decrease with $\nu$ is exponential, because of the exponentially decaying multipath intensity profile. For the frequency-flat channel, the total interference power does not depend on $\nu$; the interference is caused solely by the time variations of the channel, which cannot be counteracted by a guard interval. Again, the sum of the total interference powers resulting from the time-flat and frequency-flat limits of the channel is a very good approximation of the total interference power for the frequency-selective time-selective channel.

Figs. 6 and 7 show the effect of the sampling offset $k_{0}$ on the degradation of the SNR, for $E_{s} / N_{0}=20 \mathrm{~dB}, N=256$, and various values of $\nu$. In Fig. 6, the autocorrelation function (18) with the causal exponentially decaying multipath intensity profile has been taken. We observe that the minimum degradation occurs at $k_{0}=0$. The degradation shown in Fig. 7 corresponds to an autocorrelation function with the same Gaussian time correlation profile as in (18), but with a symmetrical exponentially decaying multipath intensity profile. The minimum degradation occurs for $k_{0} \cong \nu / 2$. Note that the optimum values of $k_{0}$, found in Figs. 6 and 7, agree with the optimum values for a time-flat channel, determined in Section V.

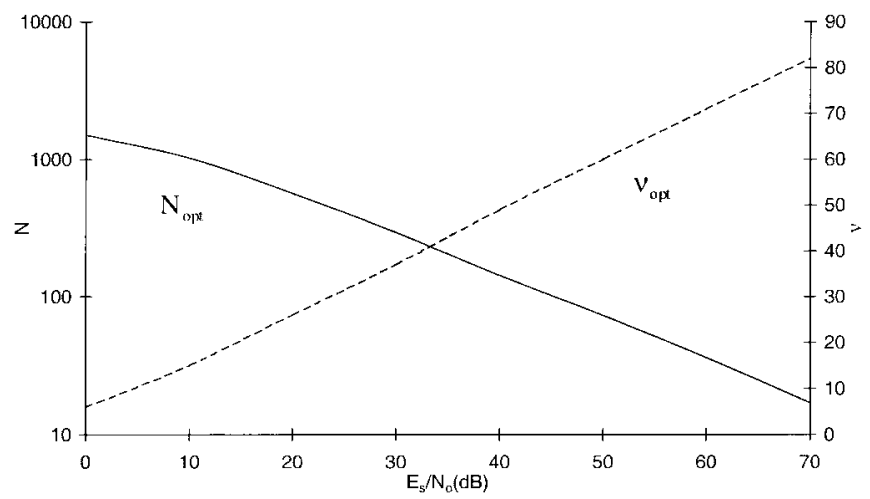

(a)

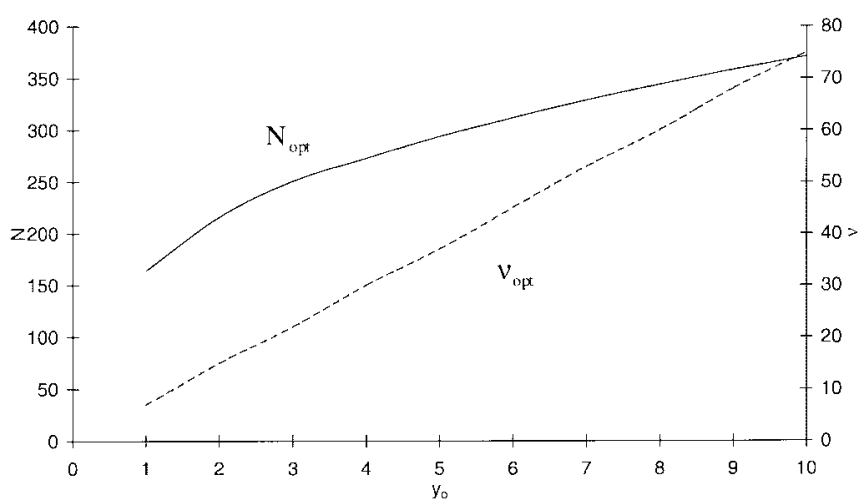

(b)

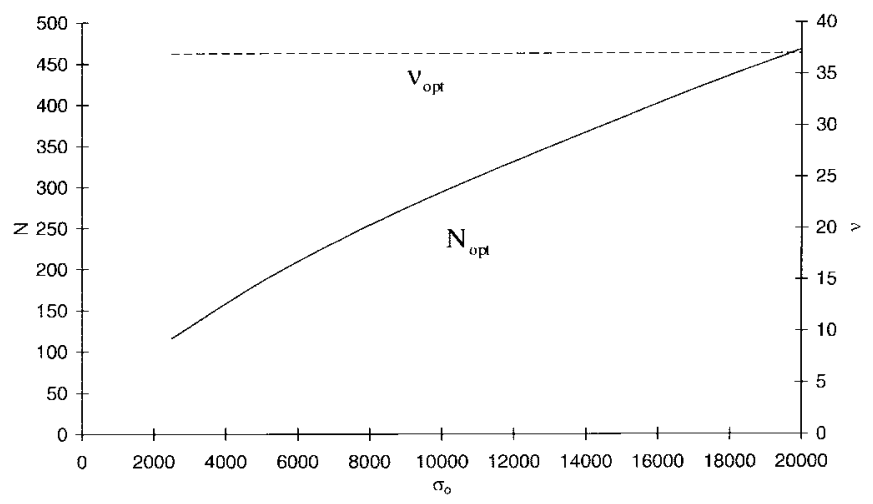

(c)

Fig. 8. Optimal system parameters.

Figs. 8 and 9 show the results from the numerical minimization of the degradation (9) with respect to the system parameters $N$ and $\nu$. As we have assumed the causal multipath intensity profile resulting from (18), the optimum timing offset $k_{0}=0$ has been selected for all considered cases.

Fig. 8(a) displays the optimum values $N_{\text {opt }}$ and $\nu_{\text {opt }}$ as a function of $E_{s} / N_{0}$, for $y_{0}=5$ and $\sigma_{0}=10000$. We observe that for increasing $E_{s} / N_{0}, N_{\mathrm{opt}}$ and $\nu_{\mathrm{opt}}$ are decreasing and increasing, respectively. This behavior can be explained as follows. For very large $E_{s} / N_{0}$, the degradation (9) converges to $-10 \log \left(P_{U} /\left(1-P_{U}\right)\right)+10 \log \left(E_{s} / N_{0}\right)$, in which case the minimization of the degradation is equivalent to the maximization of $P_{U}$. Let us denote by $\left(N_{\mathrm{opt}}(\infty), \nu_{\mathrm{opt}}(\infty)\right)$ the corresponding optimum system parameters. For very small $E_{s} / N_{0}$, the degradation (9) converges to $-10 \log \left(P_{U} N /(N+\nu)\right)$, 


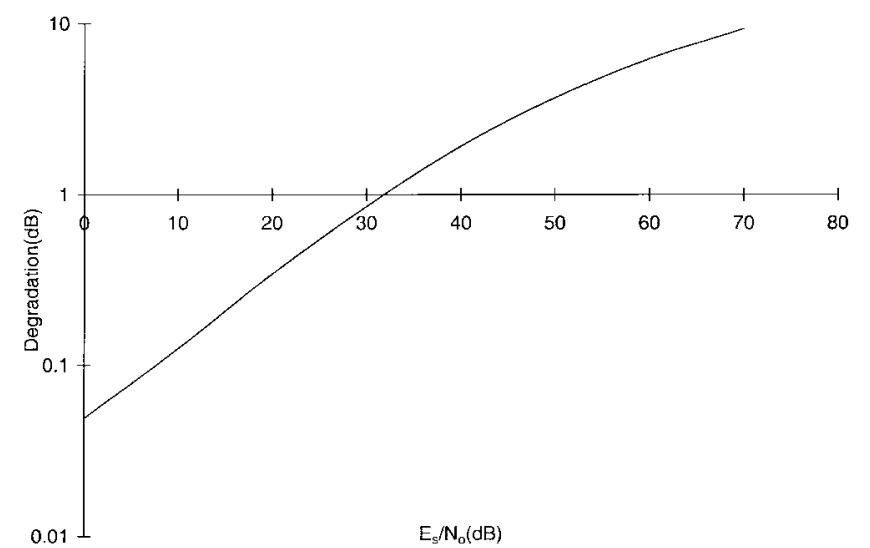

(a)

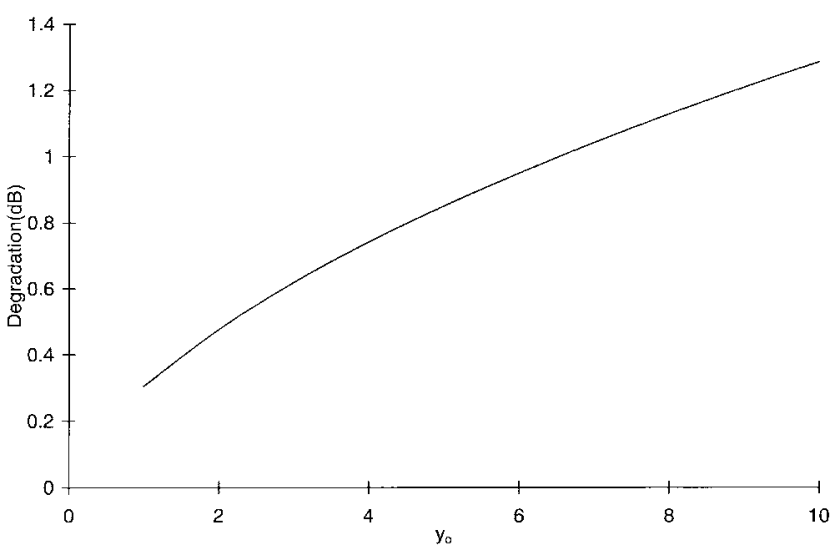

(b)

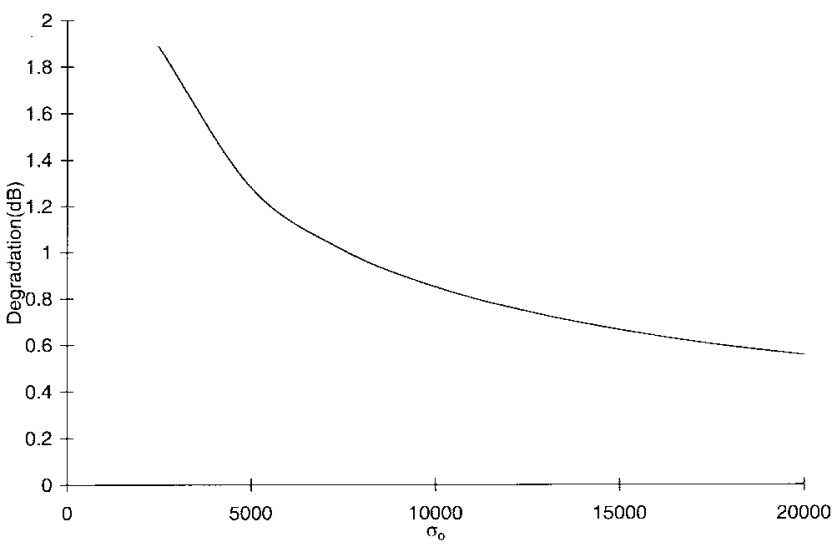

(c)

Fig. 9. Optimal degradation.

in which case the minimization of the degradation is equivalent to the maximization of $P_{U} N /(N+\nu)$. Let us denote by $\left(N_{\text {opt }}(0), \nu_{\text {opt }}(0)\right)$ the corresponding system parameters. As $N /(N+\nu)$ is decreasing with $N$ and decreasing with $\nu$, it follows that $N_{\text {opt }}(\infty)<N_{\text {opt }}(0)$ and $\nu_{\text {opt }}(\infty)>\nu_{\text {opt }}(0)$. The range of $E_{s} / N_{0}$ displayed in Fig. 8(a) is an "intermediate" range, in which $\left(N_{\mathrm{opt}}, \nu_{\mathrm{opt}}\right)$ has reached neither its limit $\left(N_{\text {opt }}(0), \nu_{\text {opt }}(0)\right)$ for low $E_{s} / N_{0}$ nor its limit $\left(N_{\text {opt }}(\infty), \nu_{\text {opt }}(\infty)\right)$ for high $E_{s} / N_{0}$. Fig. 9(a) shows the minimum degradation, corresponding to the optimum values $\left(N_{\text {opt }}, \nu_{\text {opt }}\right)$ from Fig. 8(a). This minimum degradation increases with $E_{s} / N_{0}$. As the considered range of $E_{s} / N_{0}$ is "intermediate", the above-mentioned limits of the degradation for very low and very high $E_{s} / N_{0}$ are not reached in Fig. 9(a).

Fig. 8(b) shows the optimum system parameters $\left(N_{\text {opt }}\right.$, $\nu_{\text {opt }}$ ) as function of $y_{0}$ (which is proportional to the delay spread), for $E_{s} / N_{0}=30 \mathrm{~dB}$ and $\sigma_{0}=10000$. When $y_{0}$ increases, $\nu_{\text {opt }}$ varies in proportion to $y_{0} . N_{\text {opt }}$ also increases with $y_{0}$, in order to compensate for the power efficiency reduction caused by the increase of $\nu_{\mathrm{opt}}$. However, as increasing $N_{\text {opt }}$ enhances the interference caused by the time variations of the channel, the increase of $N_{\text {opt }}$ is not linear with $y_{0}$. The minimum degradation, corresponding to $\left(N_{\mathrm{opt}}, \nu_{\mathrm{opt}}\right)$ from Fig. 8(b), increases with $y_{0}$, as can be verified from Fig. 9(b).

Fig. 8(c) shows the optimum system parameters $\left(N_{\text {opt }}, \nu_{\text {opt }}\right.$ ) as a function of $\sigma_{0}$ (which is proportional to the coherence time), for $E_{s} / N_{0}=30 \mathrm{~dB}$ and $y_{0}=5$. The optimum guard time duration $\nu_{\mathrm{opt}}$ does not depend on the coherence time, because the guard interval has no impact on the interference caused by the time-selectivity. The optimum value $N_{\text {opt }}$ is a compromise between the following phenomena.

1) Increasing $N$ reduces both the power efficiency loss and the interference caused by the frequency-selectivity.

2) Decreasing $N$ reduces the interference caused by the time-selectivity.

Hence, $N_{\text {opt }}$ increases with $\sigma_{0}$. Fig. 9(c) displays the minimum degradation corresponding to $\left(N_{\mathrm{opt}}, \nu_{\mathrm{opt}}\right)$ from Fig. 8(c); this degradation decreases with $\sigma_{0}$.

\section{CONCLUSION}

In this paper, we have first investigated the effect of the number of carriers $N$ and the guard time duration $\nu$ on the performance of an OFDM system operating on a frequencyselective time-selective fading channel. Our main conclusions are the following.

- For short frames, the time-selectivity of the channel can be ignored. The frequency-selectivity of the channel yields equal portions of ISI and ICI. The total interference power decreases with $\nu$ and is proportional to $1 / N$.

- For long frames, the frequency-selectivity of the channel can be ignored. The time-selectivity of the channel yields ICI but no ISI. The ICI power does not depend on $\nu$ and increases with $N$.

- The total interference power for a channel with both frequency-selectivity and time-selectivity is well approximated by adding the total interference powers that result from the time-flat limit and the frequency-flat limit of the considered channel. The computation of this approximated total interference power is much faster that the computation of the correct total interference power.

Further, we have determined the following optimum values of the timing offset $\left(k_{0}\right)$, the number of carriers $(N)$, and the guard time duration $(\nu)$ that minimize the degradation of the SNR, caused by ISI and ICI.

- The optimum timing offset is determined mainly by the guard time duration $\nu$ and the multipath intensity profile $R(k ; 0)$. Assuming $R(0 ; 0) \geq R(k ; 0)$, the optimum 


$$
E\left[\left|\gamma_{\ell, n, i}\left(k_{0}\right)\right|^{2}\right]=\frac{1}{N^{2}}\left(\sum_{q=0}^{\nu} \sum_{k, k^{\prime}=0}^{N-1}+\sum_{q=\nu}^{N+\nu-1} \sum_{k, k^{\prime}=q-\nu}^{N-1}+\sum_{q=-(N-1)}^{-1} \sum_{k, k^{\prime}=0}^{N+q-1}\right) e^{j 2 \pi\left(\left(\left(k-k^{\prime}\right)(n-\ell)\right) / N\right)} R\left(q-k_{0}-i(N+\nu) ; k-k^{\prime}\right) .
$$

$$
w(q ; r)=\frac{1}{N} \begin{cases}N-|r|, & 0 \leq q \leq \nu, \quad 0 \leq|r| \leq N \\ N-q+\nu-|r|, & \nu \leq q \leq N+\nu, \quad 0 \leq|r| \leq N-q+\nu \\ N+q-|r|, & -N \leq q \leq 0, \quad 0 \leq|r| \leq N+q \\ 0, & \text { elsewhere. }\end{cases}
$$

timing offsets are $k_{0}=0$ for a causal profile and $k_{0}=\nu / 2$ for a symmetric profile.

- The optimum number of carriers increases with the delay spread and the coherence time, but decreases with $E_{s} / N_{0}$.

- The optimum guard time duration increases with the delay spread and with $E_{s} / N_{0}$, but is independent of the coherence time.

- The resulting minimum degradation of the SNR increases with $E_{s} / N_{0}$ and the delay spread, but decreases with the coherence time.

\section{APPENDIX A}

Let us consider the calculation of the component $E\left[\left|\gamma_{\ell, n, i}\left(k_{0}\right)\right|^{2}\right]$. Substituting in (7) the new summation variable $q=k-m$ and considering the WSSUS channel (2), we obtain (A1), shown at the top of the page. After substituting in (A1) the summation variable $r=k-k^{\prime}$ and defining the weight function $w(q ; r)$ as in (A2), shown at the top of the page, (A1) yields

$$
\begin{array}{r}
E\left[\left|\gamma_{\ell, n, i}\left(k_{0}\right)\right|^{2}\right]=\frac{1}{N} \sum_{q=-\infty}^{+\infty} \sum_{r=-\infty}^{+\infty} w(q ; r) e^{j 2 \pi((r(n-\ell)) / N)} \\
\cdot R\left(q-k_{0}-i(N+\nu) ; r\right) .
\end{array}
$$

The sum of all powers $P_{U}+P_{\mathrm{ICI}}+P_{\mathrm{ISI}}$ equals

$$
\begin{aligned}
P_{U}+P_{\mathrm{ICI}}+P_{\mathrm{ISI}} & =\sum_{i=-\infty}^{+\infty} \sum_{n=0}^{N-1} E\left[\left|\gamma_{\ell, n, i}\left(k_{0}\right)\right|^{2}\right] \\
& =\sum_{q=-\infty}^{+\infty} R\left(q-k_{0} ; 0\right)
\end{aligned}
$$

which, considering the normalization of the energy of the impulse response of the channel, reduces to 1. Using (A3), it can easily be verified that the useful power, the power of the ICI, and the power of the ISI are given by (9).

\section{APPENDIX B}

When the frame length is large as compared to the coherence time and the delay spread, the weight function $w(k ; \ell)(\mathrm{A} 2)$ can be approximated by

$$
w(k ; \ell)=w(k ; 0)-\frac{|\ell|}{N} \quad \forall k, \ell
$$

which reduces the useful power $P_{U}$ (9) to

$$
\begin{aligned}
P_{U}=\frac{1}{N} & \sum_{\ell=-\infty}^{+\infty}\left(1-\frac{|\ell|}{N}\right) \tilde{R}(\ell) \\
& \quad-\frac{1}{N} \sum_{\ell=-\infty}^{+\infty} \sum_{k=-\infty}^{+\infty}(1-w(k ; 0)) R\left(k-k_{0} ; \ell\right)
\end{aligned}
$$

defining $\tilde{R}(\ell)$ as

$$
\tilde{R}(\ell)=\sum_{m=-\infty}^{+\infty} R(m ; \ell)
$$

For large $N$, the first contribution of (B2) behaves inversely proportional to $N$, while the second contribution behaves inversely proportional to $N^{2}$. In addition, if the guard interval is of the order of the delay spread, the second contribution is negligible as compared to the first contribution. The useful power therefore can be obtained using expression (9) where the autocorrelation function is substituted by (B3), which corresponds to the autocorrelation function of a frequency-flat fading channel. In a similar way, it can be found that the ICI power and the ISI power for large $N$ converge to values that correspond to a channel autocorrelation function $\tilde{R}(\ell) \delta(k)$.

\section{REFERENCES}

[1] G. Santella, "Bit error rate performances of M-QAM orthogonal multicarrier modulation in presence of time-selective multipath fading," in Proc. ICC'95, Seattle, WA, June 1995, pp. 1683-1688.

[2] A. Chini, M. S. El-Tanany, and S. A. Mahmoud, "On the performance of a coded MCM over multipath Rayleigh fading channels," in Proc. ICC'95, Seattle, WA, June 1995, pp. 1689-1694.

[3] N. Morinaga, N. Nakagawa, and R. Kohno, "New concepts and technologies for achieving highly reliable and high capacity multimedia wireless communication systems," IEEE Commun. Mag., vol. 38, pp. 34-40, Jan. 1997.

[4] R. Steele, Mobile Radio Communications. London, U.K.: Pentech, 1992.

[5] K. Pahlavan and A. H. Levesque, Wireless Information Networks. New York: Wiley, 1995, ch. 6. 
[6] J. A. C. Bingham, "Multicarrier modulation for data transmission, an idea whose time has come," IEEE Commun. Mag., vol. 31, pp. 5-14, May 1990.

[7] I. Kalet, "The multitone channel," IEEE Trans. Commun., vol. 37, pp. 119-124, Feb. 1989.

[8] H. Sari, G. Karam, and I. Jeanclaude, "Transmission techniques for digital terrestrial TV broadcasting," IEEE Commun. Mag., vol. 36, pp. 100-109, Feb. 1995.

[9] H. Steendam and M. Moeneclaey, "Guard time optimization for OFDM transmission over fading channels," in Proc. IEEE 4th Symp. Communications and Vehicular Technology (SCVT'96), Oct. 1996, pp. 42-48.

[10] F. Tufvesson and T. Maseng, "Optimization of sub-channel bandwidth for mobile OFDM systems," in Proc. Multiaccess, Mobility and Teletraffic-Advances in Wireless Networks (MMT), 1998, pp. 103114.

[11] M. Russel and G. L. Stüber, "Terrestrial digital video broadcasting for mobile reception using OFDM," Wireless Pers. Commun., vol. 2, pp. 45-66, 1995.

[12] J. S. Chow, J. C. Tu, and J. M. Cioffi, "A discrete multitone transceiver system for HDSL applications," IEEE J. Select. Areas Commun., vol. 9, pp. 895-908, Aug. 1991.

[13] P. S. Chow, J. C. Tu, and J. M. Cioffi, "Performance evaluation of a multichannel transceiver system for ADSL and VHDSL services," IEEE J. Select. Areas Commun., vol. 9, pp. 909-919, Aug. 1991.

[14] B. Sklar, "Rayleigh fading channels in mobile digital communication systems, part I: Characterization," IEEE Commun. Mag., vol. 38, pp. 90-100, July 1997.

[15] B. Sklar, "Rayleigh fading channels in mobile digital communication systems, Part II: Mitigation," IEEE Commun. Mag., vol. 38, pp. 102-109, July 1997.

[16] T. Pollet, H. Steendam, and M. Moeneclaey, "Performance degradation of multi-carrier systems caused by an insufficient guard interval duration," in Proc. Int. Workshop CWAS'97, Budapest, Oct. 1997, pp. 265-270.

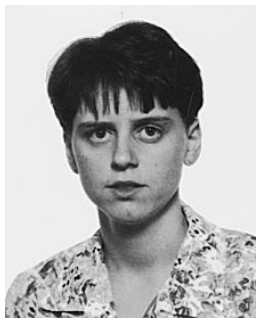

Heidi Steendam received the M.Sc. degree in electrical engineering from the University of Gent, Gent, Belgium, in 1995.

Currently, she is with the Department of Telecommunications and Information Processing, University of Gent, as a researcher and is toward the $\mathrm{Ph} . \mathrm{D}$. degree. Her main research interests include statistical communication theory, carrier and symbol synchronization, bandwidth-efficient modulation and coding, spread spectrum (multicarrier spreadspectrum), satellite and mobile communication

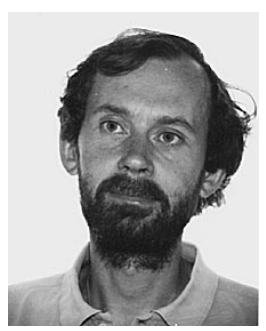

Marc Moeneclaey (M'93-SM'99) received the diploma of electrical engineering and the Ph.D. degree in electrical engineering from the University of Gent, Gent, Belgium, in 1978 and 1983 respectively.

Currently, he is with the Communications Engineering Laboratory, University of Gent, as a Researcy Director for the Flemish Fund for Scientific Research, and as a part-time Professor. His main research interests are in statistical communication theory, carrier and symbol synchronization, bandwidth-efficient modulation and coding, spread spectrum, satellite and mobile communication. $\mathrm{He}$ is the author of more than 150 scientific papers in international journals and conference proceedings. Together with Prof. H. Meyr (RWTH Aachen) and Dr. S. Fechtel (Siemens AG), he co-authored the book, Digital Communication Receivers-Synchronization, Channel Estimation, and Signal Processing (New York: Wiley, 1998).

From 1992 to 1994, he served as the Editor for Synchronization for the IEEE TRANSACTIONS ON COMMUNICATIONS. 